\title{
Hydrodynamic Synchronisation of Model Microswimmers
}

\author{
V. B. Putz and J. M. Yeomans \\ The Rudolf Peierls Centre for Theoretical Physics, \\ Oxford University, 1 Keble Road, Oxford OX1 3NP, U.K.
}

(Dated: November 4, 2018)

\begin{abstract}
We define a model microswimmer with a variable cycle time, thus allowing the possibility of phase locking driven by hydrodynamic interactions between swimmers. We find that, for extensile or contractile swimmers, phase locking does occur, with the relative phase of the two swimmers being, in general, close to 0 or $\pi$, depending on their relative position and orientation. We show that, as expected on grounds of symmetry, self T-dual swimmers, which are time-reversal covariant, do not phase-lock. We also discuss the phase behaviour of a line of tethered swimmers, or pumps. These show oscillations in their relative phases reminiscent of the metachronal waves of cilia.
\end{abstract}




\section{INTRODUCTION}

The process of movement through a fluid poses particular challenges to micron-scale swimmers, as their motion corresponds to the low Reynolds number regime, where viscous forces dominate over inertia. A swimmer moves by a cyclic deformation of its body and, at zero Reynolds number, the resultant flow field is described by the Stokes equations which have no time dependence. This kinematic reversibility of the flow means that a swimmer's deformation must be non-reciprocal in time to produce motion [1]. Non-reciprocal strokes used by biological swimmers include rotating flagella, waving cilia and surface deformations [2, [3].

A number of model swimmers have been devised which satisfy the requirement for nonreciprocal deformations. These include swimmers made of joined rods [1, 4], spheres with prescribed tangential velocities [5], rigid dumbbells with phantom flagella [6], and linked spheres undergoing prescribed shape changes [7, 8, 9, 10] or subject to periodic forces [11, 12, 13. As a result of analytical and numerical work on the model systems the swimming behaviour and efficiency of single swimmers is now rather well characterised. Moreover, experiments approximating the simple models are now becoming feasible using, for example, colloids manipulated in optical traps [14]. Much less is currently understood about the way in which the hydrodynamic interactions between two or more swimmers affect their motion. Pooley et al [15] have recently shown that the relative phase of two model swimmers may be key in determining the way in which they interact. Numerical work on large numbers of swimmers [6, 16, 17] qualitatively reproduces the instabilities seen in experiments [18, 19, 20, 21, but links between the parameters describing individual swimmers and the details of their collective behaviour are lacking.

The model swimmers which have been defined thus far in the literature complete their stroke in a fixed time. This means that the relative phase of two swimmers cannot be changed dynamically. However, given the importance of relative phase in controlling swimmerswimmer interactions, it is feasible that biological swimmers could exploit phase changes to control their relative motion. Indeed, there is evidence that hydrodynamic phase synchronisation is feasible at low Reynolds number. Perhaps the most striking example of this is the co-ordinated motion of beating cilia, or metachronal waves, which is thought to be a consequence of hydrodynamic interactions [22, 23, 24, 25]. Moreover Taylor [26] demon- 
strated that two undulating sheets minimise their dissipation if they oscillate in phase and simulations have demonstrated that sperm cells adjust their position to synchronise their motion [27]. Reichert and Stark [28] showed that two rigid helices anchored in harmonic traps rotate in phase due to hydrodynamic interactions.

Our aim in this paper is to demonstrate that low Reynolds number swimmers can phaselock as a result of the hydrodynamic interactions between them. To do this we generalise one of the simplest model swimmers, the linear three-sphere swimmer [7], to allow a variable cycle period. We find that, for extensile or contractile swimmers, phase locking does occur, with the relative phase of the two swimmers being, in general, close to 0 or $\pi$, depending on their relative position and orientation. We show that, as expected on grounds of symmetry, self T-dual swimmers, which are time-reversal covariant, do not phase-lock. We also discuss the phase behaviour of a line of tethered swimmers, or pumps. These show oscillations in their relative phases reminiscent of the metachronal waves of cilia.

In Sec. II we define an extension to the linear three-sphere swimmer which is designed to allow a variable cycle time. Sec. III describes the corresponding equations of motion and the numerical algorithm, based on the Rotne-Prager-Yamakawa approximation to zero Reynolds number hydrodynamics, we use to solve them. Sec. IV]demonstrates phase locking for two collinear swimmers, and the more complex behaviour of three collinear swimmers is described in Sec. $\mathrm{V}$ and compared to that of tethered swimmers, or pumps, in Sec VI. In Sec. VII we consider two swimmers moving in a plane and show that they can lock-in to strokes with phase difference $\sim 0$ or $\pi$ depending on their relative positions.

\section{MODEL}

We consider the linear, three-sphere swimmer illustrated in Fig. 1 [7]. The swimmer is made up of three spheres of radius $a$ joined by thin rods of extended length $D$. The lengths of the rods are altered to produce the swimming motion.

The swimming cycle proceeds in four distinct stages. First the left-hand leg is retracted $\left(I \rightarrow I I\right.$ in Fig. 1) by a distance $\xi_{r}$. This results in hydrodynamic interactions between the beads which move the swimmer a short distance to the left. Then the right leg is retracted by $\xi_{f}(I I \rightarrow I I I)$ moving the swimmer to the right. The next two steps $(I I I \rightarrow I V \rightarrow I)$ extend the left-hand and right-hand leg in sequence, back to a length $D$, and produce a 


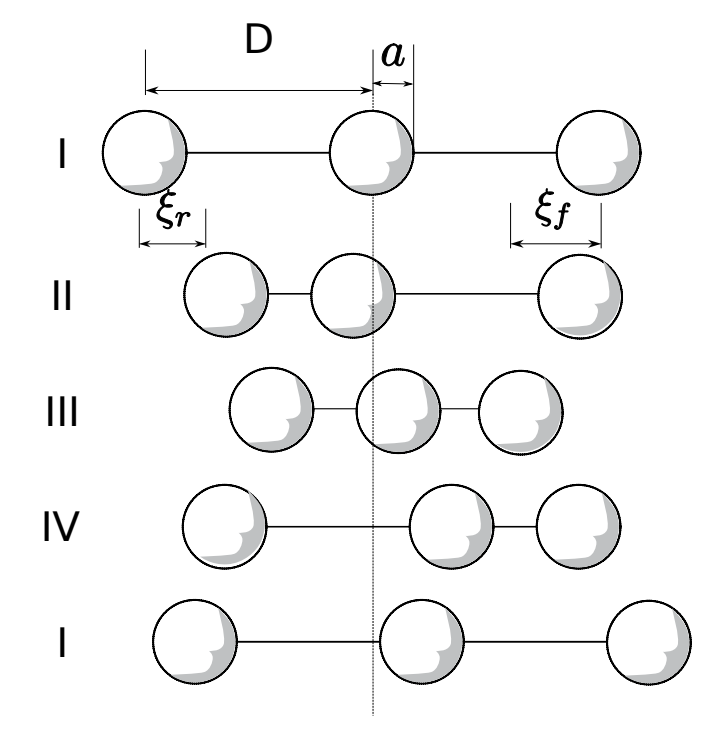

Figure 1: Cycle of the linear three-sphere swimmer; the nonreciprocal stroke permits motion at zero Reynolds number.

rightwards, followed by a leftwards motion. Because the steps which give motion to the right are performed with the passive (constant length) leg contracted, the hydrodynamic interactions between beads are stronger and hence the net motion is in this direction.

The motion of the spheres can be defined to take place at constant velocity, and we shall term this a constant velocity swimmer [8, 15, 29]. However it is also possible to drive the extension or contraction of each rod by equal and opposite, constant forces $F_{a}$ acting on the spheres at the end of that rod (a constant force swimmer). In this case, to ensure that within any given step of the cycle the length of the passive leg is unchanged, equal and opposite constraint forces must be defined to act at its ends.

Note that, for both the constant velocity and the constant force swimmers, we have defined each stage of the cycle to continue until the active rod has reached a specified length, rather than lasting for a specified time. This is an irrelevant distinction for a single swimmer because the relative position of the beads during the cycle, rather than the speed with which it completes the stroke, defines the swimmer motion; however, for more than one swimmer, the two definitions are not equivalent because hydrodynamic interactions between swimmers can affect how quickly a swimmer progresses through its swimming stroke. Interacting constant velocity swimmers have, by definition, a fixed cycle period. Interacting constant force swimmers, however, can reach a specified leg length at different times, allowing the 
possibility of phase synchronisation.

In general, the time-averaged far flow field around a swimmer is dipolar, $\sim r^{-2}$, where $r$ is the distance from the swimmer. This reflects the constraint that there are no external forces acting on the swimmer; it is an active system, producing its own energy, For the linear, three-sphere swimmer the long distance flow field is dipolar as long as $\xi_{r} \neq \xi_{f}$. For $\xi_{r}<\xi_{f}$, the swimmer is contractile (a puller), pulling in fluid at its ends and pumping it out at the sides. Conversely, for $\xi_{r}>\xi_{f}$, the swimmer is extensile (a pusher), and fluid is drawn in from the sides and pushed out the ends. For the special case $\xi_{r}=\xi_{f}$, the additional symmetry of the swimmer leads to a time-averaged flow field which has quadrupolar symmetry and which decays as $r^{-3}$ at long distances [15].

\section{EQUATIONS OF MOTION}

At sufficiently low Reynolds number that the inertial terms in the Navier-Stokes equations can be neglected the fluid velocity $\mathbf{u}$ obeys the Stokes equations

$$
\begin{gathered}
\mu \nabla^{2} \boldsymbol{u}-\nabla p=0, \\
\nabla \cdot \boldsymbol{u}=0,
\end{gathered}
$$

where $\mu$ is the dynamic viscosity and $p$ is the pressure. Because the Stokes equations are linear it is possible to write the velocity field generated by an array of spheres as a linear combination of the force $\boldsymbol{f}_{m}$ acting on each sphere $m$ [30]. This can be evaluated at the location of a sphere $n$ to give its velocity

$$
\dot{\boldsymbol{q}}_{n}=\sum_{m} \mathcal{H}_{n m} \boldsymbol{f}_{m}
$$

The tensor $\mathcal{H}_{m n}$ is known exactly only for point spheres. We use the Rotne-PragerYamakawa approximation [31, 32]

$$
\begin{aligned}
& \mathcal{H}_{m m}=(1 / 6 \pi \mu a) \mathcal{I}, \\
& \mathcal{H}_{m n}=\left(1 / 8 \pi \mu r_{m n}\right)\left\{\begin{array}{c}
{\left[\mathcal{I}+\left(\boldsymbol{r}_{m n} \otimes \boldsymbol{r}_{m n} / r_{m n}^{2}\right)\right]} \\
+\left(2 a^{2} / r_{m n}^{2}\right)\left[\frac{1}{3} \mathcal{I}-\left(\boldsymbol{r}_{m n} \otimes \boldsymbol{r}_{m n} / r_{m n}^{2}\right)\right]
\end{array}\right\}
\end{aligned}
$$

where $\boldsymbol{r}_{m n}$ is the vector between the two spheres, $m$ and $n$. This is expected to be a good approximation for $r_{m n} \gtrsim 2 a[32$. 


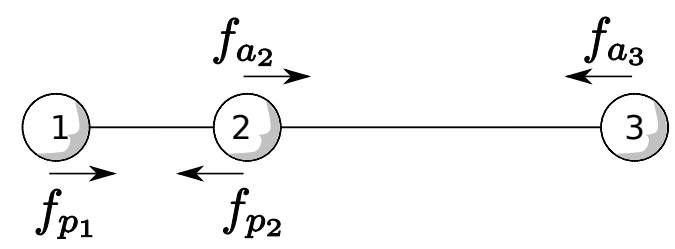

Figure 2: Forces on a three-sphere swimmer during contraction of the right-hand leg.

In general Eqn. (3) must be solved numerically. The inputs are the forces acting on the spheres bounding the active leg together with the constraint that the passive leg does not change its length. The outputs are the velocities of the beads, which in turn define the swimming motion. Imposing a fixed length on the passive leg is equivalent to imposing constraint forces on the beads at its ends. These must be equal and opposite as they are forces internal to the swimmer. In two or more dimensions, additional constraint forces are also needed to preserve the linearity of the swimmers.

To obtain an expression for the constraint forces, we define the positions of the $3 N$ spheres needed to construct $N$ swimmers by the $9 N$-component vector $\boldsymbol{q}$. We define $\boldsymbol{f}_{a} \equiv$ $\left(\boldsymbol{f}_{a 1}, \boldsymbol{f}_{a 2} \ldots\right)$ as the $9 N$-component vector listing the (known) forces moving the active legs of the swimmers and vectors $\boldsymbol{f}_{p \alpha} \equiv\left(\boldsymbol{f}_{p 1}, \boldsymbol{f}_{p 2} \ldots\right)$ describing the forces needed to impose each of the constraints which preserve the lengths of the passive legs. For example, for swimmer one, when the leg between beads 1 and 2 remains at a constant length, $\boldsymbol{f}_{p \alpha}=\left(\boldsymbol{f}_{p 1}, \boldsymbol{f}_{p 2}, 0,0,0 \ldots\right)$ (see Fig. 2). Each constraint $\alpha$ is specified by a function $C_{\alpha}(\boldsymbol{q})=0 . \dot{C}_{\alpha}=\sum_{i} \frac{\partial C_{\alpha}}{\partial q_{i}} \dot{q}_{i}=0$, or, defining $j_{\alpha i} \equiv \frac{\partial C_{\alpha}}{\partial q_{i}}$,

$$
\boldsymbol{j}_{\alpha}^{T} \cdot \dot{\boldsymbol{q}}=0
$$

The constraint forces must do no work. Therefore

$$
\boldsymbol{f}_{p \alpha}^{T} \cdot \dot{\boldsymbol{q}}=0
$$

Eqns. (6) and (7) imply that

$$
\boldsymbol{f}_{p \alpha}=\lambda_{\alpha} \boldsymbol{j}_{\alpha}
$$

Substituting Eqn. (8) into Eqn. (3), and using Eqn. (6) gives

$$
-\boldsymbol{j}_{\beta}^{T} \mathcal{H} \boldsymbol{f}_{a}=\sum_{\alpha} \boldsymbol{j}_{\beta}^{T} \mathcal{H} \boldsymbol{j}_{\beta} \lambda_{\alpha}
$$

a set of simultaneous equations that can be solved for $\lambda_{a}$. In this case $\boldsymbol{j}_{\beta}^{T} \mathcal{H} \boldsymbol{j}_{\alpha}$ is symmetric, 
and hence

$$
\lambda_{\alpha}=-\left(\boldsymbol{j}_{\alpha}^{T} \mathcal{H} \boldsymbol{j}_{\beta}\right)^{-1}\left(\boldsymbol{j}_{\alpha}^{T} \mathcal{H} \boldsymbol{f}_{a}\right)
$$

\section{PHASE LOCKING OF ONE-DIMENSIONAL SWIMMERS}

The motion of each swimmer is controlled by fixed applied forces $\boldsymbol{f}_{a}$ which act until the active arm reaches a prescribed length. When more than one swimmer is present external advection produced by fluid flow from other swimmers' motions can have an impact on how quickly the swimmer proceeds through its cycle. This allows for the possibility of phaselocking.

To discuss the notion of phase in a non-sinusoidal context, and for a variable-length cycle, it is most convenient to define a spatial phase which is a function only of the lengths of a swimmer's legs. During a complete swimming stroke, the active spheres move a total distance $2\left(\xi_{f}+\xi_{r}\right)$ relative to the centre sphere. We define the spatial phase $\phi$ of a swimmer as the fraction of this distance travelled since the beginning of the stroke multiplied by $2 \pi$, giving a number varying from 0 at the beginning of a stroke to $2 \pi$ at the completion of a stroke.

As a first example we consider the behaviour of two collinear swimmers, which will move in one dimension. Two extensile swimmers, with $\xi_{f}=0.1$ and $\xi_{r}=0.5$, were placed along the $x$-axis with their centres of mass separated by $3 D$. The initial phase of the leading swimmer was taken to be $\phi=0$ and several simulations were run for different initial phases of the trailing swimmer. The simulations were run for 50,000 swimmer cycles.

Fig. 3(a) compares the variation of the phase of the rear swimmer, measured at the beginning of the lead swimmer's stroke, with time. It is apparent that the phase difference between the two swimmers slowly drifts toward a stable value, indicating phase-locking behaviour. At the lock-in point the swimmers are approximately out of phase with each other $(\phi \approx \pi)$.

As the relative phase changes the transport of the swimmer is affected. The evolution of the distance between the two swimmers is plotted in Fig. 3(b). As the trailing swimmer changes its phase it moves closer to the lead swimmer for a time before settling, at the locking point, to motion away from the lead swimmer. This is in agreement with the conclusion in [15] that extensile, collinear, constant period swimmers attract if they are in phase but repel 


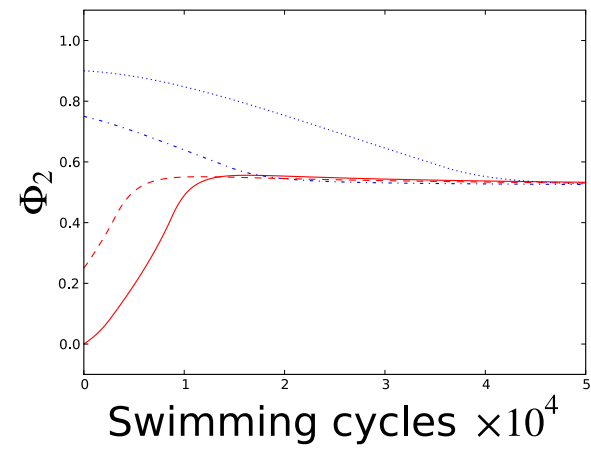

(a)

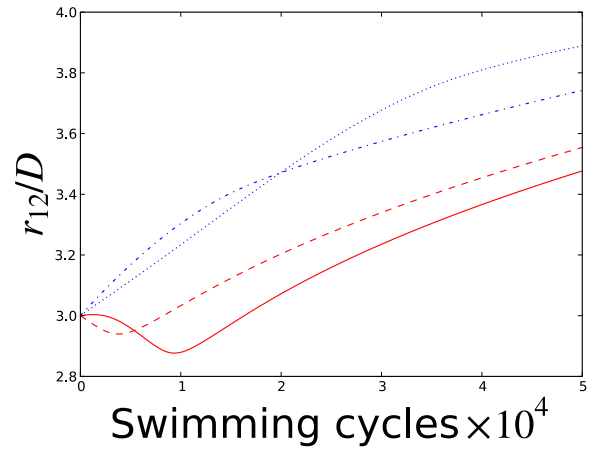

(b)

Figure 3: Time evolution of the motion of two collinear, extensile swimmers with $D=1, a=$ $0.03, \xi_{r}=0.5, \xi_{f}=0.1$ for different initial relative phase. (a) Variation of the relative phase with time: the graph shows the phase of the rear swimmer, $\phi_{2}$, measured at the beginning of the stroke of the leading swimmer $\left(\phi_{1}=0\right)$. (b) Distance between the centres of mass of the swimmers.

if they are out of phase.

The phase locking occurs because velocity gradients across a swimmer due to hydrodynamic interactions between swimmers can either assist or hinder the leg motion. Fig. 4 illustrates this by showing the change in phase experienced by the trail swimmer after one cycle. If the trail swimmer is iterating through its cycle more quickly than the lead swimmer, it will show a positive change in phase; this corresponds to moving along the graph in Fig. 4 to the right. Similarly, if the trail swimmer is hindered in its cycle, its phase will fall behind that of the lead swimmer, corresponding to moving to the left. Locking will occur when a stable point (zero phase shift) is reached. The further the curve from the $x$-axis, the faster the phase-locking behaviour proceeds. Details of the shape of the phase-change curve in Fig. 4 depend on the parameters of the swimmers and their separation. In general it becomes flatter, and more centred about a phase change of zero, as the distance between the swimmers increases, because the hydrodynamic interactions between them decrease.

By examining the points at which the curve crosses the $x$-axis for increasing swimmerswimmer spacing, we can identify how the relative phase of the swimmers in the phase-locked state varies with their separation. This is shown in Fig. 5. For a collinear arrangement of two swimmers, the lock point depends only weakly on the distance between them, although the time it takes to adjust phases increases with separation. For the case of collinear swimmers 


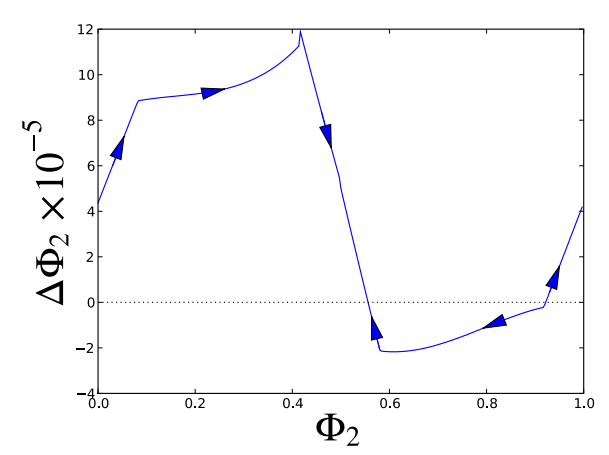

(a)

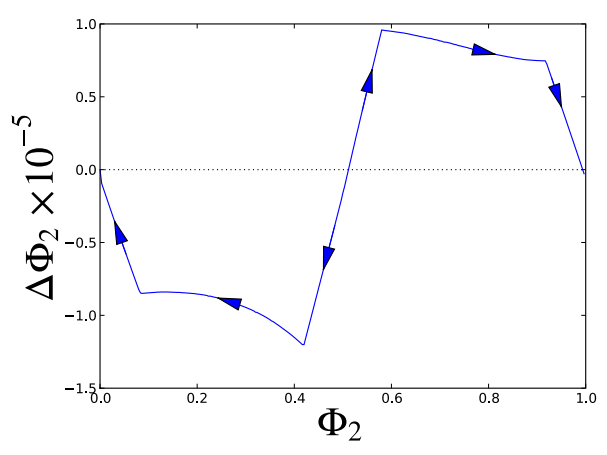

(c)

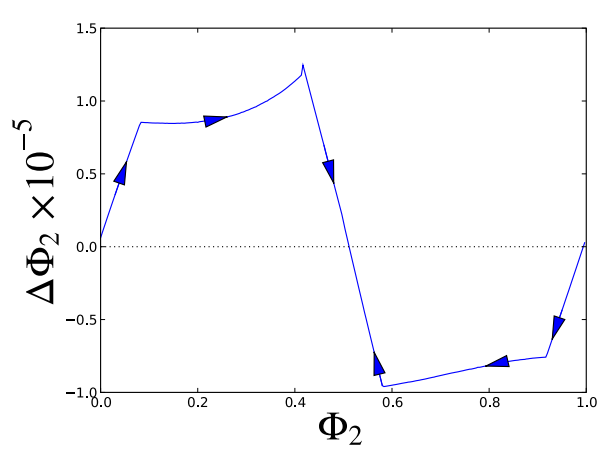

(b)

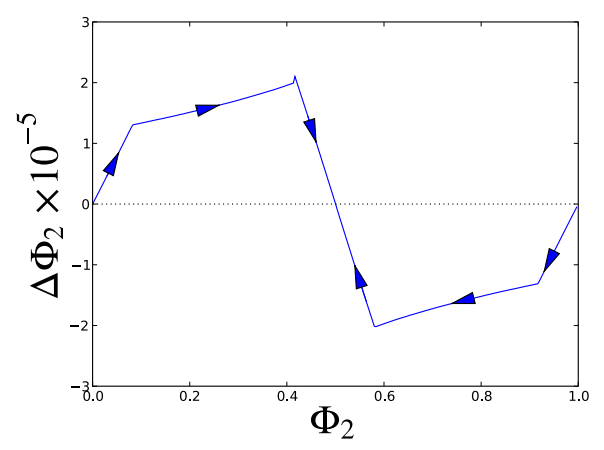

(d)

Figure 4: Change in the phase of the trailing swimmer after one swimming cycle as a function of its phase at the beginning of the cycle of swimmer 1 . Crossing points on the $x$-axis represent either an unstable (if the slope is positive) or a stable lock-in point (if the slope is negative). Arrows indicate how the swimmer's motion evolves with time. All swimmers have $\xi_{r}=0.5$, $\xi_{f}=0.1, D=1$. (a) separation $=3 D$; extensile, $\xi_{r}=0.5, \xi_{f}=0.1$; swimmers moving in same direction; (b) separation $=5 D$; extensile, $\xi_{r}=0.5, \xi_{f}=0.1$; swimmers moving in same direction; (c) separation $=5 D$; contractile, $\xi_{r}=0.1, \xi_{f}=0.5$; swimmers moving in same direction; (d) separation $=5 D$; extensile, $\xi_{r}=0.5, \xi_{f}=0.1$; swimmers moving away from each other.

moving in opposite directions (Fig $4(\mathrm{~d})$ ) the symmetry of the arrangement forces the stable lock point to be exactly halfway through the cycle $\left(\phi_{2}=\pi\right)$.

The swimmer we have considered so far has leg amplitudes $\xi_{f}<\xi_{r}$. Such an extensile swimmer is related to a contractile swimmer, with $\xi_{f}$ and $\xi_{r}$ interchanged, through a T-dual transformation ie time reversal, together with a relabelling of front and rear [33]. In terms of Fig. 4 this corresponds to an inversion of the curve $y \rightarrow-y$ (compare Figs. 4(b) and (c)). 


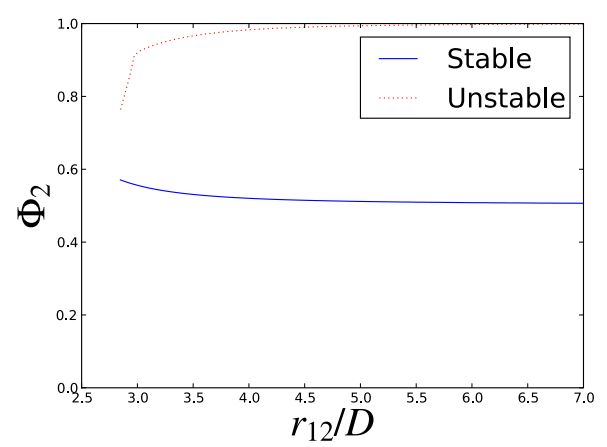

Figure 5: Stable and unstable lock-in points of the relative phase of two collinear swimmers as a function of their separation.

Hence the stable zeros of an extensile swimmer become the unstable zeros of its T-dual, contractile counterpart, and contractile swimmers lock-in to a relative phase $\phi \sim 0$.

When $\xi_{r}=\xi_{f}$, the swimmer is neither extensile nor contractile and it maps onto itself under the T-duality transformation (its flow field is not dipolar, but decays more rapidly, as $\left.r^{-3}[15]\right)$. The phase-change curve now lies along the $x$ axis, corresponding to a difference in cycle time of zero for all relative phases. Hence there is no phase-locking, as expected on grounds of symmetry, for a swimmer which is self T-dual.

\section{THREE COLLINEAR SWIMMERS}

We have demonstrated that hydrodynamic phase locking is possible for two collinear swimmers. We now investigate the behaviour of three swimmers, again moving in one dimension. Consider three collinear, extensile swimmers, initially with spacing $2.5 \mathrm{D}$, and all initially with $\phi=0$. Fig. 6 shows the phases of the middle and rear swimmer when the lead swimmer reaches the beginning of its cycle as a function of the number of swimmer cycles. It is apparent from this figure that there is no simple phase locking. Early in the simulation, the phases of swimmers 2 and 3 relative to that of swimmer 1 do not tend to a fixed point as for the two-swimmer case, but vary periodically.

As the simulation proceeds, the hydrodynamic interaction between the swimmers acts to change their relative positions. Swimmers 1 and 2 remain at a similar spacing, and swimmer 3 drifts away as shown in Fig. 6(b). The stronger interaction between swimmers 1 and 2 constrains their relative phase to be close to $\pi$, but with a small oscillation imposed by the 


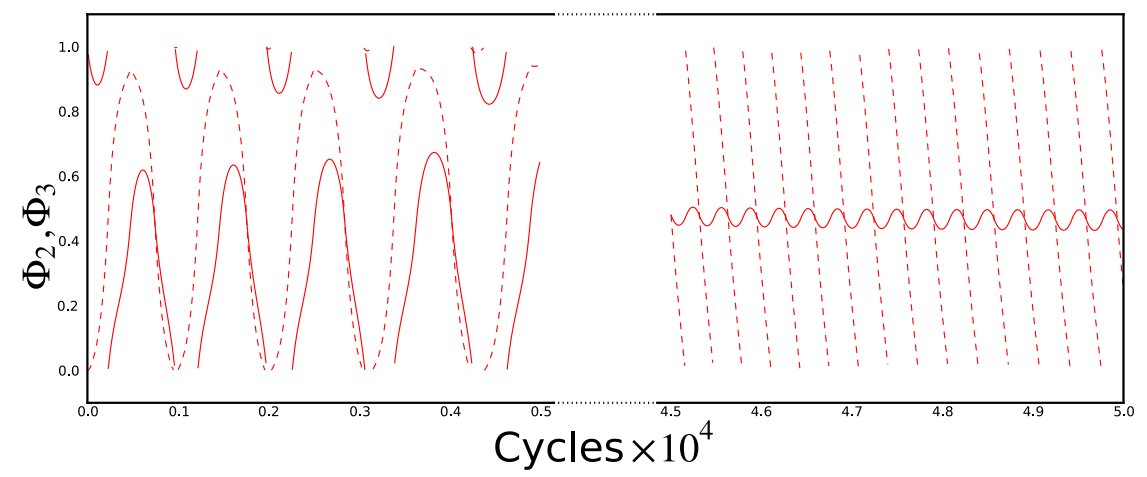

(a)

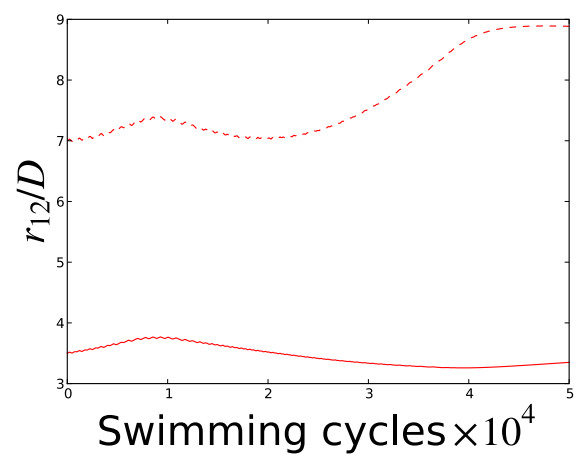

(b)

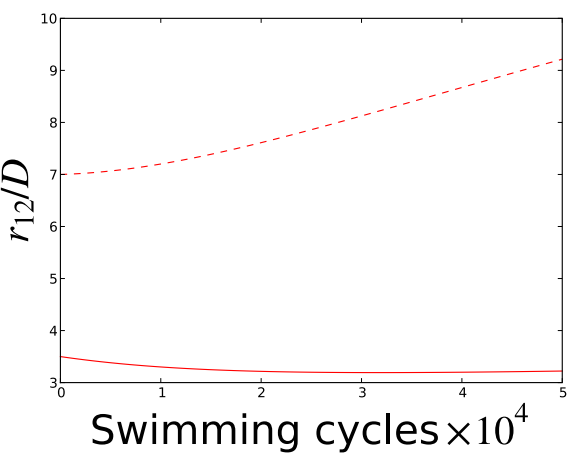

(c)

Figure 6: Time evolution of the relative phase of three collinear swimmers. (a) relative phase of the middle and rear swimmers at the beginning of the front swimmer's cycle (b) displacements of the middle and rear swimmers from the front swimmer in a constant force swimmer simulation (c) displacements of the middle and rear swimmers from the front swimmer in a constant velocity swimmer simulation.

third swimmer, as its phase decreases at an approximately constant rate.

For comparison we present, in Fig 6(c), similar results for the relative positions of constant velocity swimmers which remain in phase. There is the same tendency for one of the swimmers to drift away from the other two. At the longest time this swimmer is still moving away, whereas for the constant force case, its position has been stabilized by the changing relative phase. 


\section{TETHERED COLLINEAR SWIMMERS}

Swimmers which are constrained to remain in a fixed position become pumps that drive a net flow field. The flow field and hydrodynamic interactions will be changed by the constraint; in particular the far flow field will, in general, decay as $r^{-1}$ because of the force holding the swimmer in position. The locking is likely to be simpler, as the distance between the pumps, and hence the strength of the hydrodynamic interactions between them, will remain constant.

To investigate this we considered collinear swimmers, whose centre of mass was fixed in space by applying a small external force to each sphere at each time step of the simulation. The force necessary to do this was relatively small, $\sim 10^{-3}$ of the magnitude of the internal forces necessary to move the legs.

The evolution of the relative phases of the pumps with time is shown in Fig. 7. Two extensile pumps phase-lock with a phase difference of $\pi$, as for the free swimmers. Three or four equispaced pumps show stable oscillations in their relative phases. These are reminiscent of the metachronal waves observed in arrays of cilia [22, 23, 24, 25].

\section{TWO DIMENSIONS}

The phase-locking behaviour of two swimmers is considerably more complex when they are not collinear. To investigate this we ran simulations with two extensile swimmers, initially parallel, for different distances apart and for different angles $\theta$ between the swimmer orientation and the vector joining their centres. For each position, we ran the swimmers through one cycle at relative phases from 0 to $2 \pi$ to produce phase-change curves analogous to those in Fig. 4. Examples are shown in Fig. 8, together with a central panel summarising the locking behaviour as a function of swimmer spacing and $\theta$.

As $\theta$ varies, the phase change curves change dramatically. Consider first increasing $\theta$ along the arc $G D B$ in Fig. 8. At $G(\theta=0)$, there is lock-in to a phase difference $\phi=\pi$, corresponding to the the swimmers oscillating in antiphase. As $\theta$ increases, the fixed point loses its stability via a bifurcation and two new stable fixed points appear $(D)$. These move apart and annihilate, such that stability is transferred to the fixed point at $\phi=0$, and the swimmers synchronize in phase $(B)$. 


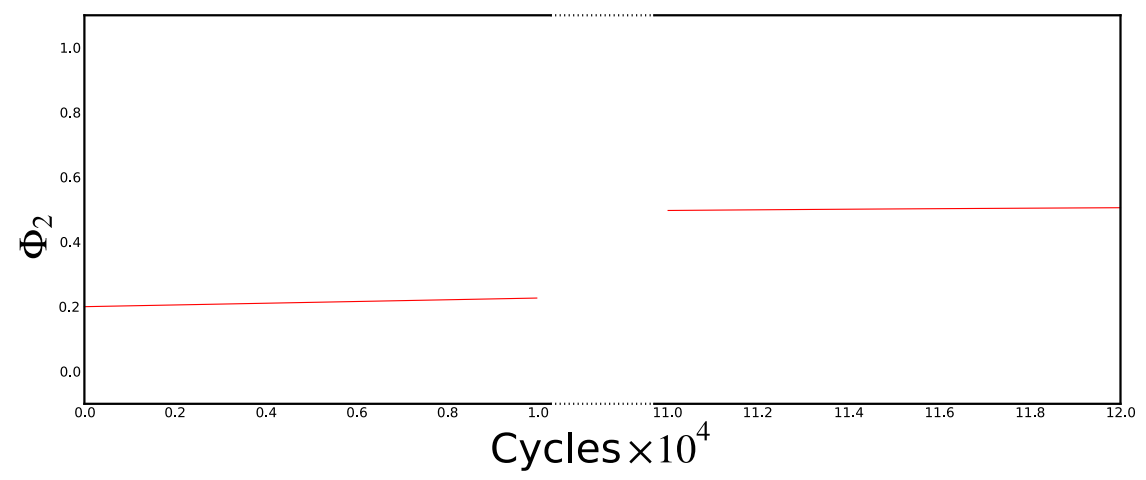

(a)

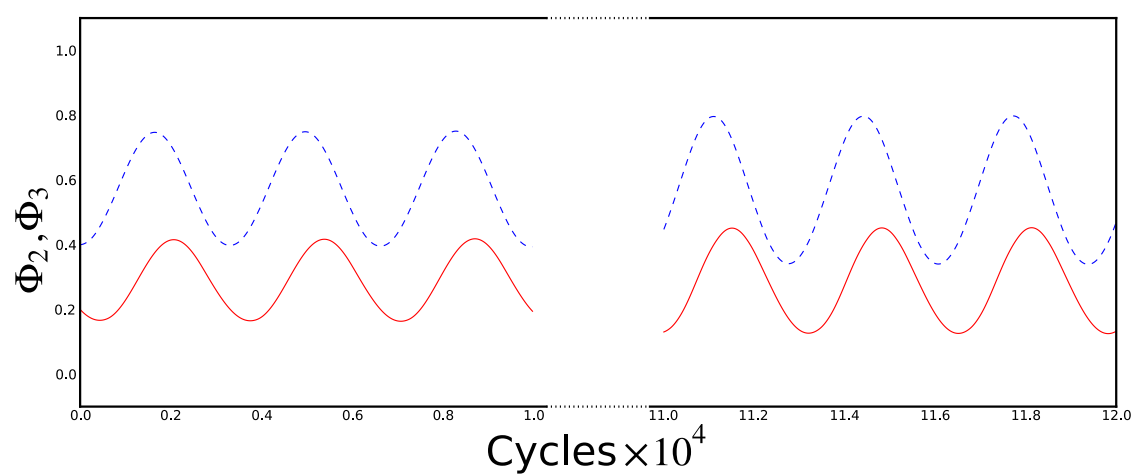

(b)

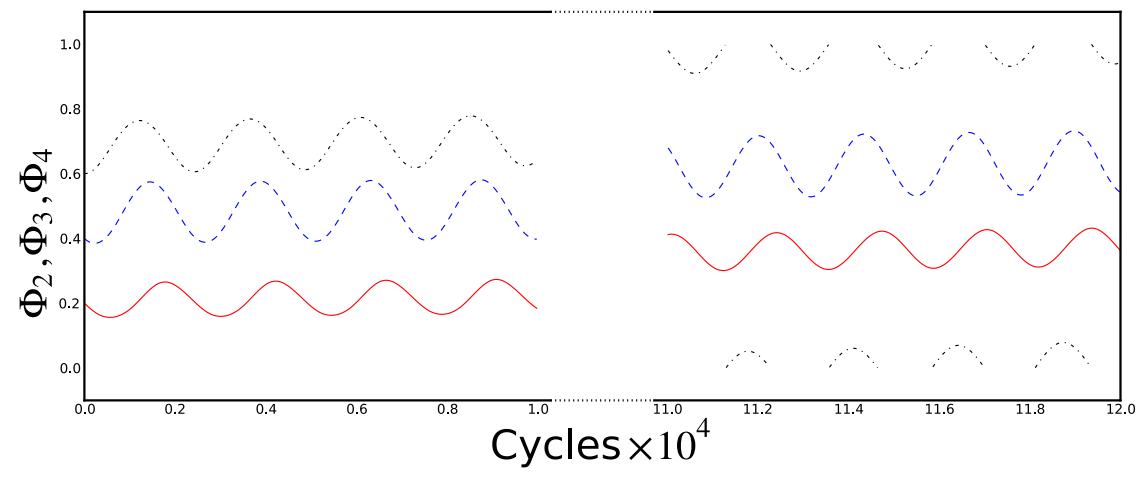

(c)

Figure 7: Time evolution of the relative phases of collinear pumps with time (a) two (b) three (c) four swimmers. The phases are measured at the beginning of the cycle of pump 1

This interchange of stability occurs several times as $\theta$ varies, giving regions of lock-in which are alternately in-phase and out-of-phase. The pattern reflects the dipolar symmetry of the hydrodynamic interactions.

As the swimmers move further apart, remaining parallel but at the same angle $\theta$, (e.g. 

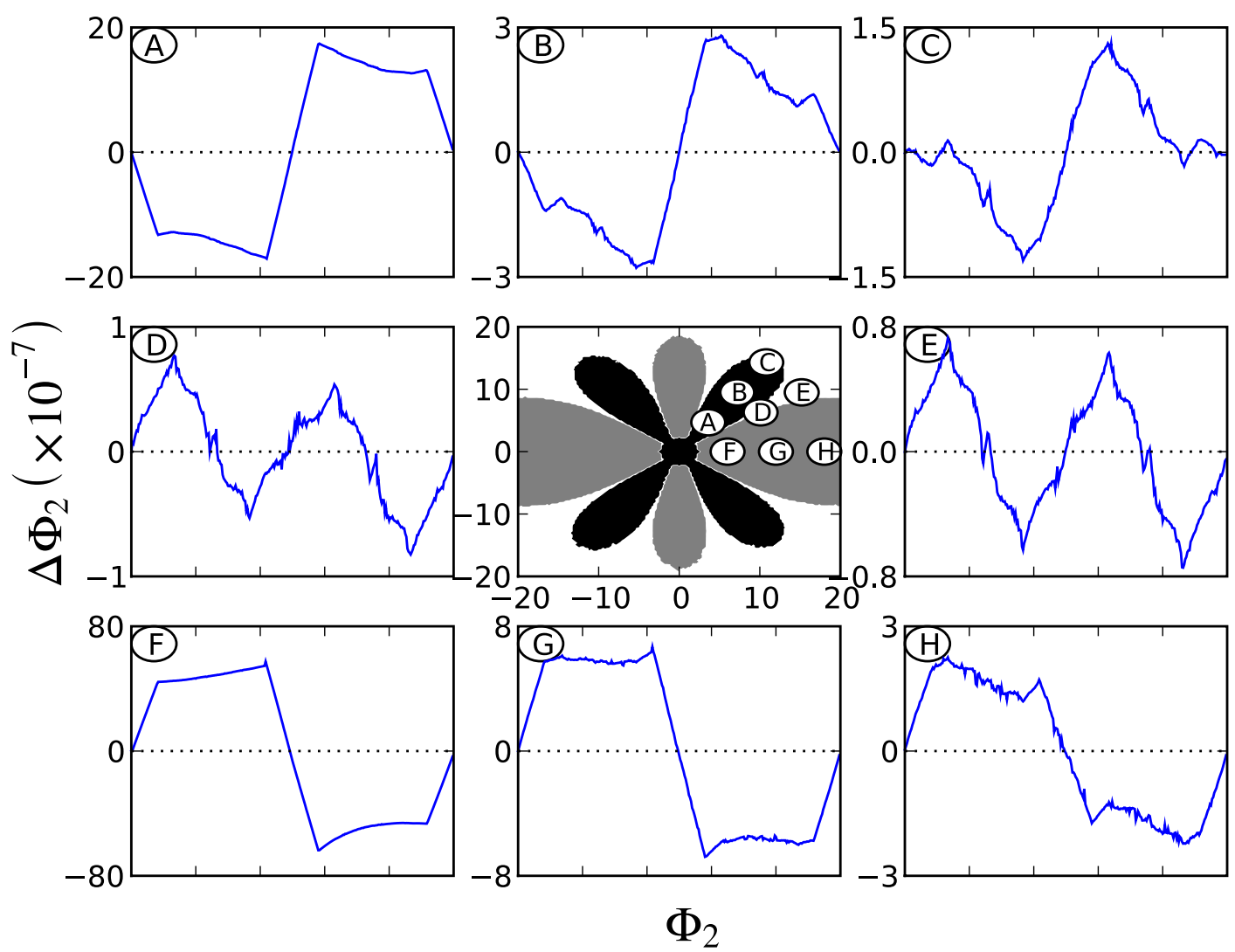

Figure 8: Centre: phase locking behaviour of two extensile swimmers. The swimmers are parallel and swimmer 1 is at the origin. The axes are labelled in units of $D$. When swimmer 2 lies in the grey areas, the swimmers synchronise out-of-phase $(\phi=\pi)$. When swimmer 2 lies in the black areas, the swimmers synchronise in-phase $(\phi=0)$. The white areas represent regions with two or more stable zeros. Sides: phase change curves corresponding to swimmer configurations $A, B \ldots H$. In each case the change of phase of swimmer 2 per cycle is plotted as a function of the phase of swimmer 2 measured at the beginning of the stroke of swimmer 1 .

FGH in Fig. 8), the curves retain approximately the same shape but the change in phase per cycle is smaller, reflecting the weakening interactions. Beyond the shaded regions two or more lock points are seen but it is difficult to distinguish locking from numerical noise. 


\section{DISCUSSION}

We have extended the definition of a simple linear, three-sphere swimmer model to permit variable stroke periods, and hence to allow for the possibility of phase synchronisation at zero Reynolds number. We find phase locking for two swimmers, and more general cooperative phase behaviour for more than two swimmers. The phase-locking is driven by hydrodynamic interactions.

In general the swimmers lock-in to a phase difference of $\sim 0$ or $\sim \pi$, depending on their relative positions, although more complicated synchronisation behaviour is possible. For three swimmers the relative phases oscillate, together with a superimposed drift in time, as the swimmer positions vary. The locking is slow, tens or hundreds of swimmer cycles, becoming slower with increasing separation.

A similar synchronisation is seen for tethered swimmers (pumps), although the behaviour is simpler because the relative positions of the pumps do not vary. Two collinear pumps lock-in to a phase difference 0 or $\pi$ for contractile or extensile flow fields respectively. Three or four reach a limit cycle, with relative phases oscillating in a way reminiscent of cilia.

We note that self T-dual swimmers and pumps, such as the three-sphere swimmer with equal arm lengths, are forbidden by symmetry from phase locking. This agrees with the result of Kim and Powers [34] who showed that two rigid helices with parallel axes, driven by the same torque, do not synchronise.

Phase synchronisation in the form of metachronal waves, which are thought to be stabilised by hydrodynamic interactions, is well established in cilliary dynamics. It would be interesting to see whether any similar behaviour is apparent in systems of biological swimmers. Moreover, phase-locking will affect the behviour of fabricated micropumps, and questions remain as to whether synchronisation can enhance flow.

\section{ACKNOWLEDGEMENTS}

We thank G.P. Alexander, J. Dunkel, S.A. Edwards, C.M. Pooley, and I. Zaid for helpful discussions. 


\section{BIBLIOGRAPHY}

[1] E. M. Purcell, Am. J. Phys. 45, 3 (1977).

[2] M. Silverman and M. Simon, Nat. 249, 73 (1974).

[3] J. Lighthill, SIAM Rev. 18, 161 (1976).

[4] L. E. Becker, S. A. Koehler, and H. A. Stone, J. Fluid Mech. 490, 15 (2003).

[5] T. Ishikawa, M. P. Simmonds, and T. J. Pedley, J. Fluid Mech. 568, 119 (2006).

[6] J. P. Hernandez-Ortiz, C. G. Stoltz, and M. D. Graham, Phys. Rev. Lett. 95, 204501 (2005).

[7] A. Najafi and R. Golestanian, Phys. Rev. E 69, 062901 (2004).

[8] D. J. Earl, C. M. Pooley, J. F. Ryder, I. Bredberg, and J. M. Yeomans, J. Chem. Phys. 126, 064703 (2007).

[9] R. Dreyfus, J. Baudry, and H. A. Stone, Euro. Phys. J. B. 47, 161 (2005).

[10] J. E. Avron, O. Kenneth, and D. H. Oaknin, New J. Phys. 7, 234 (2005).

[11] E. E. Keaveny and M. R. Maxey, Phys. Rev. E 77, 041910 (2008).

[12] E. Gauger and H. Stark, Phys. Rev. E 74, 021907 (2006).

[13] B. U. Felderhof, Phys. Fluids 18, 063101 (2006).

[14] M. Leoni, J. Kotar, B. Bassetti, P. Cicuta, and M. C. Lagomarsino, Soft Matter pp. 472-476 (2009).

[15] C. M. Pooley, G. P. Alexander, and J. M. Yeomans, Phys. Rev. Lett. 99, 228103 (2007).

[16] J. P. Hernandez-Ortiz, P. T. Underhill, and M. D. Graham, arXiv:0811.1814v1 [physics.flu-dyn] (2008).

[17] T. Ishikawa, J. T. Locsei, and T. J. Pedley, J. Fluid Mech. 615, 401 (2008).

[18] D. Guell, H. Brenner, R. Frankel, and H. Hartman, J. Theor. Biol. 135, 525 (1988).

[19] N. H. Mendelson, A. Bourque, K. Wilkening, K. R. Anderson, and J. C. Watkins, J. Bacteriol. 181, 600 (1999).

[20] C. Dombrowski, L. Cisneros, S. Chatkaew, R. E. Goldstein, and J. O. Kessler, Phys. Rev. Lett. 93, 098103 (2004).

[21] X. Wu and A. Libchaber, Phys. Rev. Lett. 84, 3017 (2000).

[22] M. C. Lagomarsino, B. Bassetti, and P. Jona, Eur. Phys. J. B 26, 81 (2002). 
[23] M. C. Lagomarsino, P. Jona, and B. Bassetti, Phys. Rev. E 68, 021908 (2003).

[24] S. Gueron, K. Levit-Gurevich, N. Liron, and J. J. Blum, Proc. Natl. Acad. Sci. USA 94, 6001 (1997).

[25] S. Gueron and K. Levit-Gurevich, Proc. Natl. Acad. Sci. USA 96, 12240 (1999).

[26] G. Taylor, Proc. R. Soc. Lond. Ser. A 209, 447 (1951).

[27] Y. Yang, J. Elgeti, and G. Gompper, Phys. Rev. E 78, 061903 (2008).

[28] M. Reichert and H. Stark, Eur. Phys. J. E 17, 493 (2005).

[29] R. Golestanian and A. Ajdari, Phys. Rev. E 77, 036308 (2008).

[30] J. G. Kirkwood and J. Riseman, J. Chem. Phys. 16, 565 (1948).

[31] J. Rotne and S. Prager, J. Chem. Phys 50, 4831 (1969).

[32] H. Yamakawa, J. Chem. Phys. 53, 436 (1970).

[33] G. P. Alexander, C. M. Pooley, and J. M. Yeomans, Phys. Rev. E 78, 045302 (2008).

[34] M. Kim and T. R. Powers, Phys. Rev. E 69, 061910 (2004). 